\title{
Incorporating remote patient monitoring in virtual pulmonary rehabilitation programs
}

\author{
Jenna V. Jangalee B.Sc., RRT, CRE, CTE ${ }^{1}$, Pooneh Ghasvareh $\mathrm{PhD}^{2}$, Jordan A. Guenette PhD ${ }^{3,4}$, Jeremy Road MD ${ }^{5}$
}

\begin{abstract}
JV Jangalee, P Ghasvareh, JA Guenette, J Road. Incorporating remote patient monitoring in virtual pulmonary rehabilitation programs. Can J Respir Ther 2021;57:83-89. doi: 10.29390/cjrt-2021-015.

Most pulmonary rehabilitation (PR) programs have had to adapt due to the COVID-19 pandemic and associated restrictions. Current alternative homebased programs have limitations and require modification. In this paper, we outline a novel method to monitor home-based PR programs, which has the potential to improve PR safety and efficacy. This new method is based on a remote patient monitoring (RPM) system with connected smart devices that enables the Respiratory Therapist (RT) to have real-time access to patient data including heart rate and peripheral oxygen saturation during exercise. The RPM system also monitors daily physical activity, sedentary time, sleep quality, rescue inhaler use, and maintenance inhaler adherence, among other variables, which has the added advantage of predicting patterns consistent with symptoms that may require medical intervention. To increase privacy, data are anonymized at all levels and only the RT has access to patient information. RPM systems have the potential to give practitioners a holistic view of the participants' health status to better evaluate them during the entire PR program and to improve self-management. As this is not a formal research study, we cannot make definitive conclusions about the efficacy of the system, and further research is needed to examine safety and to compare our approach to other ways of conducting PR.
\end{abstract}

Key Words: COVID-19; COPD; $\mathrm{SpO}_{2}$; heart rate; real-time; smart devices; pulmonary rehabilitation; remote patient monitoring

\section{INTRODUCTION}

Pulmonary rehabilitation (PR) is an essential part of the health management of people living with chronic respiratory conditions [1-3]. It is one of the most effective nonpharmacological approaches to help people maintain and maximize their level of independence and function, reduce dyspnea, improve exercise tolerance, promote a sense of well-being, decrease the number of hospitalizations [4], and reduce the use of excessive health care resources [5].

The COVID-19 pandemic has presented major challenges in how clinicians deliver care to their patients. Some PR programs around the world have been temporarily canceled due to infection control policies and physical distancing requirements, and for the immediate future, PR is unlikely to be delivered in its traditional face-to-face setting. Therefore, behavior change and self-management training should target a framework of adaptation.

There are some alternatives to outpatient in-person PR programs, such as telephone-based, home-based rehabilitation models [6-9], and video-based exercising models $[7,10]$. Some of the considerations for delivering virtual PR programs are the challenges associated with assessing exercise capacity, monitoring physiological and symptomatic responses to exercise, and patient safety [11]. The Canadian Thoracic Society has recently published guidance for resumption of PR programs during COVID-19 and introduced a number of alternatives to in-person PR [11]. These alternatives provide some ability to monitor a patient's response to exercise by using the modified Borg scale and to track exercise performance by the 1-min sit-to-stand test. However, the Borg scale is subjective and results of the sit-to-stand test cannot easily be used to prescribe exercise; therefore, some modifications are needed to deliver safe and effective virtual PR [11].

Vancouver Coastal Health developed a clinical protocol for virtual PR programs using Zoom video-conferencing for exercise training and education. The PR Team at Vancouver General Hospital (VGH) has refined the video-based exercise program by adding remote monitoring smart devices (Agartee Technology Inc. Vancouver, BC, Canada), to address some of the limitations in delivering virtual PR. This new real-time monitoring system was chosen because it can automatically collect sleep data (e.g., total sleep hours, number of awakenings, sleep efficiency) [12], physical activity (e.g., number of steps, walking duration, sedentary time), vital signs (e.g., peripheral oxygen saturation $\left(\mathrm{SpO}_{2}\right)$, heart rate $\left.(\mathrm{HR})\right)$, maintenance medication adherence, and rescue inhaler usage (e.g., number of puffs in each use, number of times used and duration of pressing to assess inhaler technique) without the need for manual data entry. Also, the method can be seamlessly integrated into virtual PR programs and it is easy to use for seniors at home. It enables the Respiratory Therapist (RT) to have access to health data of the participants over long periods of time, along with access to real-time data of their $\mathrm{HR}$ and $\mathrm{SpO}_{2}$ during the exercise sessions.

\section{METHODS}

Three PR programs at VGH have been using the remote patient monitoring (RPM) system to deliver PR. Each PR program had 4 participants ( $33 \%$ males for all three programs). The age ranges were from 58 to 80 years with a median interquartile range (IQR) of 71 (15)

\footnotetext{
${ }^{1}$ Vancouver General Hospital Pulmonary Rehabilitation Program, Vancouver, BC, Canada; ${ }^{2}$ Agartee Technology Inc., Vancouver, BC, Canada; ${ }^{3}$ Centre for Heart and Lung Innovation, Providence Health Care Research Institute and The University of British Columbia, St. Paul's Hospital, Vancouver, BC, Canada; ${ }^{4}$ Department of Physical Therapy, Faculty of Medicine, The University of British Columbia, Vancouver, BC, Canada; ${ }^{5}$ The Lung Centre at Vancouver General Hospital, Vancouver, BC, Canada

Correspondence: Jenna V. Jangalee, 899 West 12th Ave, Vancouver, BC V571M9, Canada. Tel: 778-870-5779, E-mail: Jenna.Jangalee@vch.ca
}

Published online at https://www.cjrt.ca on 22 July 2021 
years. Some patients had moderate-to-severe COPD ( $\mathrm{FEV}_{1} / \mathrm{FVC}$ ranged from 0.27 to 0.79 with a median (IQR) of $0.66(0.29)$ ).

Participants were included if they were fluent in English and had a referral from their physician along with clearance to participate in exercise. There was no need to have a background in using technological devices although each individual was required to have a laptop, PC, or tablet at home to use for the Zoom meetings. Zoom sessions were not recorded for privacy reasons and the RPM data were encrypted at all levels with data retained in Canada. Participant information was anonymized by a random six-digit identifier, and only the RT could access the patient information.

After obtaining consent, all participants were mailed the RPM devices prior to commencing their PR program. The participants were successfully able to follow the simple instructions to set up the devices and did not require any technical support.

Below are strategies that were used during the program and some of the benefits that have been observed.

\section{EQUIPMENT FROM THE SERVICE PROVIDER}

1) All of the participants received a package of connected monitoring devices as shown in Table 1. All devices were preconfigured and there was no need for recharging or set-up. A weight scale and thermometer were also included to get a more complete assessment of the participant.

2) The RT had access to a web-dashboard to monitor real-time HR and $\mathrm{SpO}_{2}$ during the exercise sessions and to monitor the historical data outside of the class. Table 2 shows the health metrics that can be collected in real-time during the whole program.

3) Optional: Participants were given access to their health data with a simple to use mobile app. These data can motivate them to maintain or improve their activity level, work on their sleep quality (e.g., have fewer awakenings at night and improve sleep efficiency), and use it as a medication usage reminder to improve adherence.

\section{PROGRAM SCHEDULE}

The program consists of 8 weeks. On weeks $1-7$, the educational and exercise sessions start. The participants continuously use the monitoring devices and the system collects their baseline sleep, activity, and vital signs after a few days. During the exercise sessions, the RT can monitor changes in $\mathrm{HR}$ and $\mathrm{SpO}_{2}$ remotely (see Exercise session section). On the last week of the program, the individuals' post-assessment starts. The historical data gathered during weeks 1-8 of the program are helpful to evaluate the improvement in activity and vital sign data.

\section{PROGRAM SESSIONS}

\section{Education session}

Each session contains a 1-h education session over Zoom. On average, each participant only missed 1.3 out of 21 sessions. The education sessions are presented by a multidisciplinary team including RTs/Certified Respiratory Educators, a Physiotherapist, an Occupational Therapist, a Registered Dietician, and a Psychologist. Topic areas are selected to provide a foundation for continued self-management including lung anatomy, physiology, pathophysiology, controlled breathing techniques, mucus clearance techniques, lung trigger avoidance, respiratory diagnostic tests, respiratory medications, home oxygen, nutrition, energy conservation, lung attacks, and action plans [13].

\section{Exercise session}

To ensure the safety of exercise sessions, participants receive a safety checklist via email prior to their initial assessment. The participants sign a waiver that they can comply with the safety checklist recommendations for pre-exercise, during exercise, and post-exercise. The safety checklist requirements are reverbalized prior to starting each exercise session. For example, if the participants are alone at home at the time of exercise, they are asked to unlock their front door as an extra safety precaution. All participants give their emergency contact and building manager information to the PR program before enrollment so that in rare cases of emergency facilitators could call 911 for help.

The exercise session is $1 \mathrm{~h}$ in length and uses a modified circuit training model incorporating both arm and leg strengthening exercises with aerobic minutes interspersed between sets. The sets, repetitions, and aerobic minutes for each participant increases over the 8 weeks of the program. During the exercise session, the RT oversees the participants on Zoom and, at the same time, can monitor the $\mathrm{HR}$ and $\mathrm{SpO}_{2}$ through a live dashboard in real-time. The participants were given education on

TABLE 1

The list of devices included in the package, their purpose, and the way to use them

\begin{tabular}{|c|c|c|c|}
\hline $\begin{array}{l}\text { Device } \\
\text { name }\end{array}$ & Image & What does it do? & When to use? \\
\hline Modem & & $\begin{array}{l}\text { Receives and transmits the data in } \\
\text { real-time to the care team }\end{array}$ & Always plug-in at home \\
\hline Wristband & & $\begin{array}{l}\text { Collects physical activity patterns } \\
\text { and sleep quality [12] }\end{array}$ & Always wear on the wrist \\
\hline $\begin{array}{c}\text { Pulse } \\
\text { Oximeter }\end{array}$ & & TracksHR and oxygen saturation & $\begin{array}{l}\text { Use at least once a day, or when feeling } \\
\text { severe or abnormal respiratory symptoms, } \\
\text { and during the exercise sessions }\end{array}$ \\
\hline $\begin{array}{l}\text { Add-on } \\
\text { Medication } \\
\text { tracker }\end{array}$ & & $\begin{array}{l}\text { Tracks the time and duration of } \\
\text { inhaled medication }\end{array}$ & $\begin{array}{l}\text { Put the sensor on inhaled medication } \\
\text { and use it as usual }\end{array}$ \\
\hline Weight scale & & Tracks weight fluctuations & Use at least once a day \\
\hline Thermometer & & Tracks body temperature and fever & Use at least once a day \\
\hline
\end{tabular}




\section{TABLE 2}

\section{List of the metrics and their trends the RT can access by the web-dashboard (both real-time and the historical trends)}

\begin{tabular}{|c|c|}
\hline Metrics & What is included in the report? \\
\hline $\begin{array}{l}\text { Probability of exacerbation/ } \\
\text { Shortness of Breath (SOB) }\end{array}$ & Increased probability of exacerbation/SOB (See discussion) \\
\hline \multirow{2}{*}{ Medication Adherence } & Adherence to inhaled medication usage \\
\hline & Number of puffs and usage duration of inhaled medication \\
\hline \multirow{3}{*}{ Activity } & Number of steps per day \\
\hline & Distance walked per minute \\
\hline & Sedentary time per day \\
\hline \multirow{5}{*}{ Sleep } & Duration of sleep per night \\
\hline & Number of awakenings per night \\
\hline & Major sleep interruptions per night (being awake more than $30 \mathrm{~min}$ ) \\
\hline & Major sleep shift (changes in sleep/awake time and duration of sleep) \\
\hline & $\begin{array}{l}\text { Sleep efficiency (Number of hours being sleep over number of hours } \\
\text { laying down, in percentage) }\end{array}$ \\
\hline Vital signs & $\begin{array}{l}\mathrm{HR}(\mathrm{bpm}), \mathrm{SpO}^{2}(\%) \text {, perfusion Index (PI\%), body temperature (C), } \\
\text { and weight }(\mathrm{lb}, \mathrm{kg})\end{array}$ \\
\hline
\end{tabular}

how to use a pulse oximeter to obtain a valid reading and thus were advised to be stationary for a brief period before each reading to ensure stability in the $\mathrm{HR}$ and $\mathrm{SpO}_{2}$ signal.

Figure 1 shows an example of the live dashboard, where the visualization of the data can enable the RT to monitor the exercise response of all the participants simultaneously and in real-time.

Each exercise session starts with $\mathrm{SpO}_{2}$ and HR monitoring of the participants to get their resting baseline (first point in Figure 1). The RT then goes through the routine of aerobic and strengthening exercises. After the third or fourth aerobic minute, vital sign monitoring is done to ensure the participants have reached the desired HR and to identify if their $\mathrm{SpO}_{2}$ level is appropriate (second point in Figure 1). The target HR is $60 \%-80 \%$ of the maximum HR based on their age [14]. The session ends with 5 min of cool-down stretches, followed by another $\mathrm{SpO}_{2}$ and HR measurement to make sure the HR is back to normal and that the client is not in an episode of desaturation before logging off the virtual exercise session (third point in Figure 1). The sampling frequency for both $\mathrm{HR}$ and $\mathrm{SpO}_{2}$ is $60 \mathrm{~Hz}$, and each data point is the average of 10 samples. The average is taken after ensuring that the data are stable. The only other parameter that is used for monitoring during the exercise session is the modified $0-10$ BORG scale to assess the intensity of dyspnea. The objective physiological endpoints of the exercise program using the live dashboard data are as follows: HR $>130$ beats per minute (or $80 \%$ max predicted HR) and/or $\mathrm{SpO}_{2}<90 \%$. Symptomatic endpoints include: BORG sustained $>5$ (signifying severe dyspnea), fatigue, leg pain, dizziness, or chest pain resembling angina.

\section{DISCUSSION}

Current methods to conduct virtual PR programs have some limitations compared with traditional in-person programs [10]. For example, monitoring the exercise responses depends on having access to pulse oximeters for self-monitoring or just relying on the BORG scale [11]. Also, the 6-minute walk test (6 MWT) and Incremental Shuttle Walk Test to assess exercise capacity cannot be administered at home according to recognized standards in most home environments [15].

The goal of the modified PR program is to address some of these limitations by:

1. Providing connected pulse oximeters to the participants so that the RT can monitor real-time $\mathrm{SpO}_{2}$ and $\mathrm{HR}$ during the exercise sessions. These data ensure that participants are safely exercising at the appropriate intensities. It also provides direct readings from the oximeter without having to rely on participant interpretation of values.

2. Giving access to complimentary sleep, physical activity, and vital sign data outside of the program, which helps the RT monitor progress and provides feedback to maximize adherence. Examples of sleep and activity data that the RT has access to include total sleep hours, number of awakenings per night, and sleep efficiency (the total sleep time over the time spent in bed at night), number of steps, duration of walking, and sedentary time per day.

3. Identifying the most at-risk participants using artificial intelligence algorithms. The RPM system notifies the RT on the web-dashboard if there is a high probability of an acute exacerbation or if the patient is experiencing increased dyspnea during their activities of daily living. This has been done by comparing the participants' data with their baseline and finding if there is a major change in sleep quality (e.g., when the time to go to bed or get up in the morning changes from the average, when the number of awakenings increases from baseline or when there is a reduction in average total sleep for consecutive nights), physical activity (e.g., when there is a reduction in average activity for consecutive days), sedentary time (e.g., when sedentary time increases from average on consecutive days) and $\mathrm{SpO}_{2}$ level, and/or the number of inhaled medication doses (e.g., when rescue inhaler usage increases or when the number of puffs per day of rescue inhaler usage at night increases). The RT can check the detailed behavioral, vital sign, and medication usage data and 


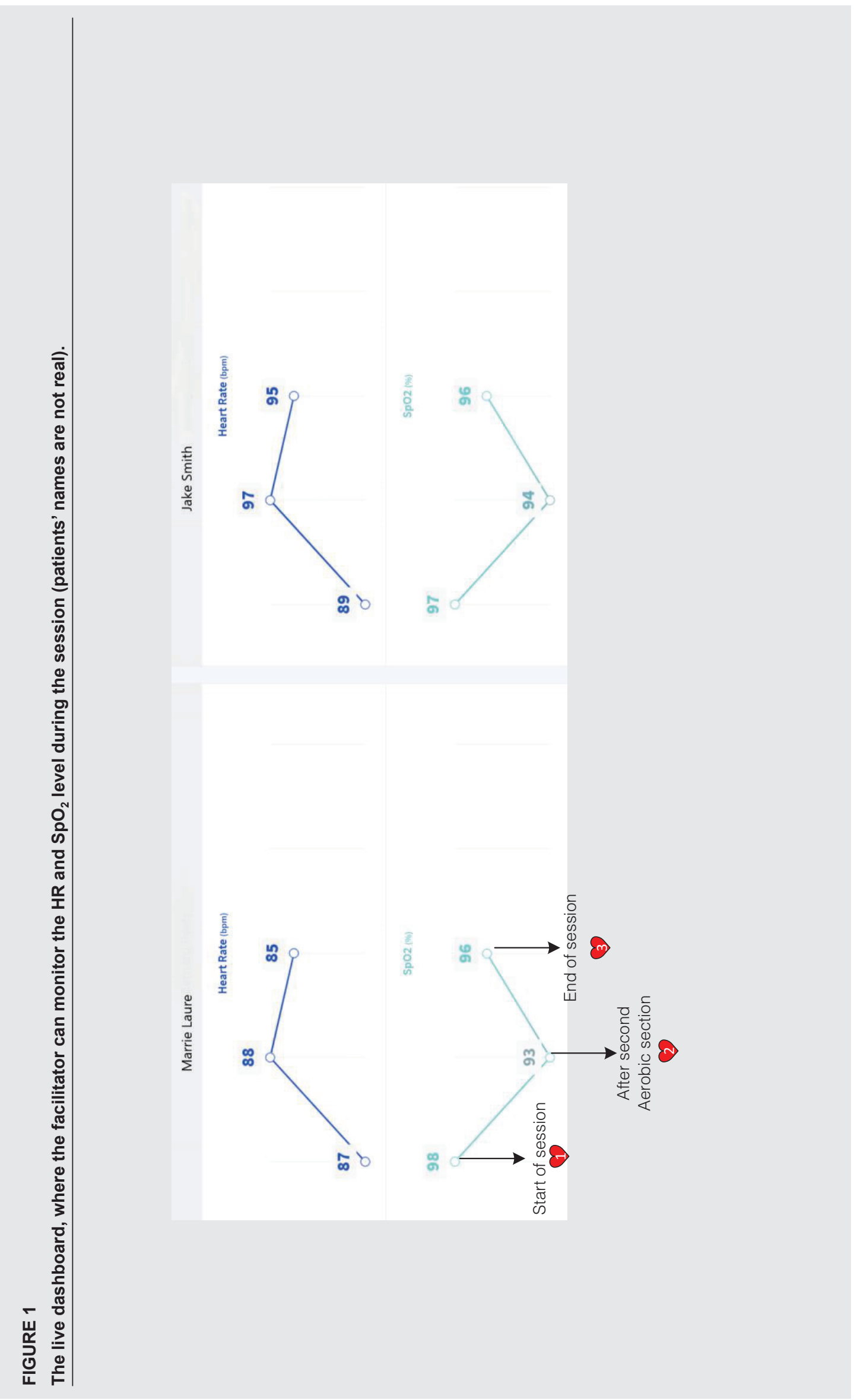




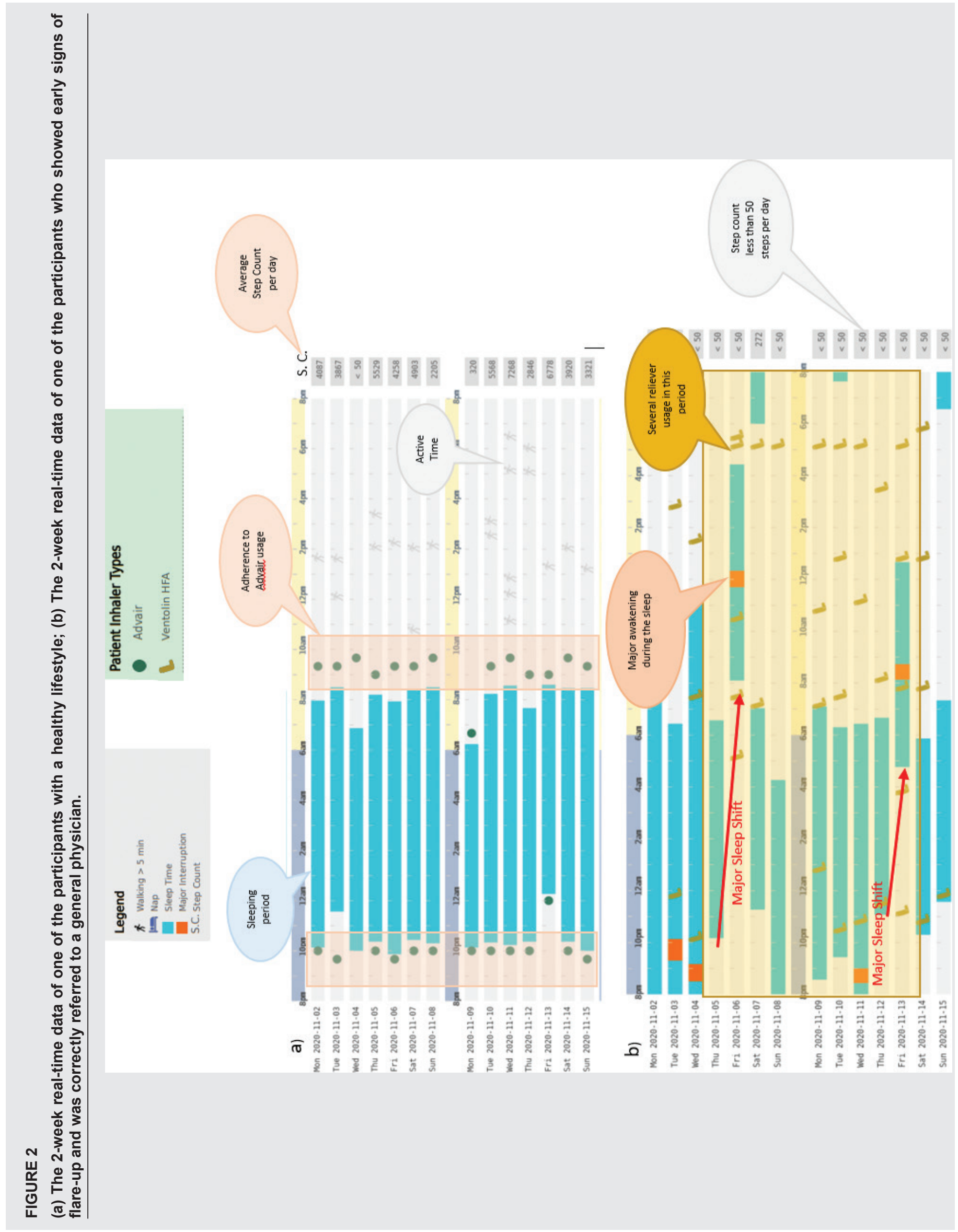


interview the participant to either promote behavior change, activate respiratory action plans, provide instructions on the best ways to use or access medication, and encourage follow-up with physicians if needed. The participant and the RT can continue to follow-up regarding the shared plan and can continue to monitor the data to track improvements.

Figure 2 shows a comparison of sleep/activity and medication usage data from: (a) a relatively healthy and stable participant with chronic obstructive pulmonary disease (COPD), (b) a participant with COPD that showed early signs of an exacerbation.

It is clear that the first participant has an active lifestyle with more than 2000 step counts per day, a regular sleep routine, and consistently uses maintenance medications. The second participant has an irregular sleep pattern, with some major sleep interruptions. The participant also used the reliever inhaler several times during the night. The implementation of this innovation can help the RT have a timely follow-up with identified at-risk participants (similar to the second participant in Figure 2), use focused questions to determine if the participant is experiencing signs and symptoms of an exacerbation, coach participants to use self-management skills to recognize these changes in symptoms, and to better utilize their action plans, which will include when to seek assessment from a general practitioner/specialist if required or when to go seek emergent care.

Some of the benefits of the system reported by the clinicians are summarized below:

1) Having access to live $\mathrm{HR}$ and $\mathrm{SpO}_{2}$ during the exercise sessions:

- ensures safety and that target HR (60\%-80\% of max. HR for age) is being met during exercise

- ensures resting $\mathrm{HR}$ and $\mathrm{SpO}_{2}$ are at safe levels at the start and end of the exercise

- helps clients to correlate BORG dyspnea ratings to $\mathrm{SpO}_{2}$ and HR

- enables the RT to virtually troubleshoot specific symptoms (e.g., dizziness)

- benefits the clients who cannot afford a personal oximeter

- increases confidence in the accuracy of values

2) Having access to the number of nighttime awakenings:

- correlates with client symptoms and determine whether they are in a potential flare-up and helps to take further action

- helps to pursue further follow-up from a physician if the client has sleep disturbances at initial assessment or scores poorly on the Epworth Sleepiness Scale

3) Having access to activity data:

- helps the RT to see the progression of exercise over time

- motivates clients to track their own activity levels and create goals using the optional mobile app

- decreased step count may correlate with respiratory worsening or flare ups of other conditions requiring assessment (i.e. arthritis)

- helps to start conversations on barriers to physical activity

4) Having access to inhaled medication tracker usage data:

- corroborates self-reported adherence to actual inhaler usage

- helps to start a conversation around adherence once a trend is noted

- helps to monitor PRN as needed use of reliever inhalers to assess whether worsening is experienced

5) Having access to weight and temperature data:

- monitors real-time weight at the beginning and end of the program

- visualizes weight trends over time if losing/gaining weight is a goal

- monitors fever which is especially useful during the COVID-19 pandemic to detect early symptoms

All participants completed the program successfully. Based on the feedback forms from two programs $(n=8)$, all participants appreciated the opportunity to attend the PR program during the pandemic. One of the participants preferred to have in-person PR if possible, and two of them said they would not have been able to attend the program if it was not performed virtually because of challenges with transportation. There are no data on the social interactions of the participants; however, some of them showed interest in continuing their communication with other participants even after the program.

There are some limitations to conducting virtual PR programs in general, and adding the RPM can minimize these limitations. For example safety of the participants during exercise can be improved by adding an objective measure to monitor physiological responses to exercises. Privacy issues regarding RPM data were minimized by anonymizing the data and keeping the data sets in Canada. Another potential limitation to virtual PR is that participants should have WI-FI connectivity for Zoom meetings. The RPM technology used with our participants works with long-term evolution (LTE) and does not need WI-FI; however, LTE connectivity can still have some issues, particularly in remote areas, which may result in some delays to see the data. Although some social aspects of in-person PR are missing, conducting virtual PR in the time of a pandemic allows some interactions in the current environment.

In the future, it is possible to expand the application of this innovation and study its effects. Below are some suggestions:

1) Using RPM for 6 MWT: The wristband provided in the package can measure the distance that the participant walks per minute and therefore could potentially be used for the $6 \mathrm{MWT}$ before and after the program if they are unable to perform the test in a more controlled clinical environment.

2) Using RPM in maintenance pulmonary rehabilitation programs: Maintenance programs have been defined as ongoing supervised exercise at a lower frequency per week than the initial pulmonary rehabilitation program [16]. Having access to historical data can significantly help the RT to evaluate the progress of the maintenance programs, ensure the participants either maintain or progress their activity levels, and follow-up with them if major changes in their behavior or vital signs occur.

\section{CONCLUSION}

Due to the COVID-19 pandemic and associated restrictions, many in-person PR programs have been canceled. Alternative home-based programs (e.g., video conferencing) need major modifications to improve safety and efficiency. The PR team at VGH has refined home-based PR programs by adding a RPM system to video-conferencing. This new method uses connected smart devices that enable RTs to have real-time access to participants' health data. The method is user friendly for seniors and can be seamlessly integrated into home-based virtual PR programs.

Implementation of this innovation has helped the RTs safely conduct the PR exercise sessions at home. The historical data of sleep, activity, vital signs and inhaled medication usage gathered during the program has also enabled the RTs to better monitor the participant's progress during the program and has helped them to intervene faster when the participants have experienced early signs of an exacerbation. Future work is needed to use RPM systems in randomized controlled trials of home-based PR to determine if they contribute to improved clinical outcomes.

\section{DISCLOSURES}

\section{Contributors}

JJ: Conceptualization, data collection, writing original draft; PG: writing original draft, JG: reviewing and editing, JR: conceptualization, reviewing and editing.

\section{Funding}

This study did not receive any funding. 


\section{Competing Interests}

All authors have completed the ICMJE uniform at www.icmje.org/coi disclosure.pdf. JJ: Declares Agartee Technology VIBE devices were trialed in the VGH Pulmonary Rehabilitation - Chronic Lung Disease Program. Payment was not received personally, by the pulmonary rehabilitation program nor by Vancouver General Hospital for its use. PG: Declares personal fees for scientific research with Agartee Technology Inc. JG: Declares that he is the Scientific Director and an Advisory Board Member for Agartee Technology Inc. JG and his wife also serve as consultants for Agartee Technology Inc. and hold shares in the company JR: Declares that he is a consultant to Agartee Technologies Inc. and holds shares in Agartee Technology Inc.

\section{REFERENCES}

1. Romero-Arenas S, Martínez-Pascual M, Alcaraz PE. Impact of resistance circuit training on neuromuscular, cardiorespiratory and body composition adaptations in the elderly. Aging Dis 2013 Oct;4(5):256. doi: 10.14336/AD.2013.0400256.

2. Mazzeo RS, Cavanagh P, Evans WJ, et al Exercise and physical activity for older adults. Med Sci Sports Exerc 1998 Jun 18;30(6):992-1008. doi: 10.1249/00005768-199806000-00033.

3. Cheng ST, Wu YK, Yang MC, et al. Pulmonary rehabilitation improves HR variability at peak exercise, exercise capacity and health-related quality of life in chronic obstructive pulmonary disease. Heart Lung 2014 May 1;43(3):249-55. doi: 10.1016/j.hrtlng.2014.03.002.

4. Ries AL, Kaplan RM, Limberg TM, Prewitt LM. Effects of pulmonary rehabilitation on physiologic and psychosocial outcomes in patients with chronic obstructive pulmonary disease. Ann Intern Med 1995 Jun 1;122(11):823-32. doi: 10.7326/0003-4819-122-11-199506010-00003.

5. Fishman AP. Pulmonary rehabilitation research. Am J Respir Crit Care Med 1994 Mar;149(3):825-33. doi: 10.1164/ajrccm.149.3.8118655.

6. Tsai LL, McNamara RJ, Moddel C, Alison JA, McKenzie DK, McKeough ZJ. Home-based telerehabilitation via real-time videoconferencing improves endurance exercise capacity in patients with COPD: the randomized controlled TeleR Study. Respirology 2017 May;22(4):699_ 707. doi: 10.1111/resp.12966.

7. Holland AE, Hill CJ, Rochford P, Fiore J, Berlowitz DJ, McDonald CF. Telerehabilitation for people with chronic obstructive pulmonary disease: feasibility of a simple, real time model of supervised exercise training. J Telemed Telecare 2013 Jun;19(4):222-6. doi: 10.1177/1357633×13487100.

8. Ghanem M, Abd ELaal E, Mehany M, Tolba K. Home-based pulmonary rehabilitation program: effect on exercise tolerance and quality of life in chronic obstructive pulmonary disease patients. Ann Thorac Med 2010 Jan;5(1):18. doi: 10.4103/1817-1737.58955.

9. Nield M, Hoo GW. Real-time telehealth for COPD self-management using Skype ${ }^{\mathrm{TM}}$. COPD 2012 Nov 29;9(6):611-9. doi: 10.3109/ 15412555.2012.708067.

10. Thomas MJ, Simpson J, Riley R, Grant E. The impact of home-based physiotherapy interventions on breathlessness during activities of daily living in severe COPD: a systematic review. Physiotherapy 2010 Jun 1;96(2):108-19. doi: 10.1016/j.physio.2009.09.006.

11. Dechman G, Aceron R, Beauchamp M, et al. Delivering pulmonary rehabilitation during the COVID-19 pandemic: A Canadian Thoracic Society position statement. Canadian.

12. Syed N, Road JD, Ryerson CJ, Guenette JA. Evaluation of the vibe actigraph in patients with chronic obstructive pulmonary disease: a pilot study. IEEE J Transl Eng Health Med 2020 Aug 20;8:1-8. doi: 10.1109/ JTEHM.2020.3018399.

13. Wilson JS, O'Neill B, Reilly J, MacMahon J, Bradley JM. Education in pulmonary rehabilitation: the patient's perspective. Archiv Phys Med Rehabil 2007 Dec 1;88(12):1704-9. doi: 10.1016/j.apmr.2007.07.040.

14. Mejia R, Ward J, Lentine T, Mahler DA. Target dyspnea ratings predict expected oxygen consumption as well as target $\mathrm{HR}$ values. Am J Respir Crit Care Med 1999 May 1;159(5):1485-9. doi: 10.1164/ajrccm.159.5.9810039.

15. Holland AE, Spruit MA, Troosters T, et al. An official European Respiratory Society/American Thoracic Society technical standard: field walking tests in chronic respiratory disease. Eur Respir J 2014 Dec 1;44(6): 1428-46. doi: 10.1183/09031936.00150314

16. Alison JA, McKeough ZJ, Johnston K, et al. Australian and New Zealand Pulmonary Rehabilitation Guidelines. Respirology 2017 May;22(4): 800-19. doi: 10.1111/resp.13025. 